\title{
Is macroturbulence in OB Sgs related to pulsations?
}

\author{
Sergio Simón-Díaz ${ }^{1,2}$, Artemio Herrero ${ }^{1,2}$, Katrien Uytterhoeven ${ }^{3}$, \\ Norberto Castro ${ }^{1,2}$, Conny Aerts ${ }^{4,5}$ and Joachim Puls ${ }^{6}$ \\ ${ }^{1}$ Instituto de Astrofísica de Canarias, E-38200 La Laguna, Tenerife, Spain; email: \\ ssimon@iac.es; ${ }^{2}$ Departamento de Astrofísica, Universidad de La Laguna, E-38205 La \\ Laguna, Tenerife, Spain; ${ }^{3}$ Laboratoire AIM, CEA/DSM-CNRS-Université Paris Diderot; CEA, \\ IRFU, SAp, centre de Saclay, 91191, Gif-sur-Yvette, France; ${ }^{4}$ Instituut voor Sterrenkunde, \\ Katholieke Universiteit Leuven, Celestijnenlaan 200D, 3001 Leuven, Belgium; ${ }^{5}$ IMAPP, \\ Department of Astrophysics, Radboud University Nijmegen, PO Box 9010, 6500 GL Nijmegen, \\ the Netherlands; ${ }^{6}$ Universitätssternwarte München, Scheinerstr. 1, 81679 München, Germany
}

\begin{abstract}
As part of a long term observational project, we are investigating the macroturbulent broadening in $\mathrm{O}$ and $\mathrm{B}$ supergiants (Sgs) and its possible connection with spectroscopic variability phenomena and stellar oscillations. We present the first results of our project, namely firm observational evidence for a strong correlation between the extra broadening and photospheric line-profile variations in a sample of $13 \mathrm{Sgs}$ with spectral types ranging from O9.5 to B8.
\end{abstract}

Keywords. stars: early-type, stars: atmospheres, stars: oscillations, stars: rotation, supergiants

\section{Introduction}

The presence of an important extra line-broadening mechanism (in addition to the rotational broadening and usually called macroturbulence) affecting the spectra of $\mathrm{O}$ and B Sgs is well established observationally (see Simón-Díaz et al. 2010, and references therein). Lucy (1976) postulated that this extra broadening may be identified with surface motions generated by the superposition of numerous non-radial oscillations. More recently, Aerts et al. (2009) computed time series of line profiles for evolved massive stars broadened by rotation and hundreds of low amplitude non-radial gravity mode oscillations and showed that the resulting profiles could mimic the observed ones. Stellar oscillations are a plausible explanation for the extra broadening in O and B Sgs, but this hyphotesis needs to be observationally confirmed.

\section{The macroturbulence - LPV connection}

As a first step, in Simón-Díaz et al. (2010), we investigated the possible connection between the macroturbulent broadening and the presence and temporal behaviour of line-profile variations (LPVs) in a sample of 11 late-O and early-B Sgs, 2 late B-Sgs, and 2 late-O, early-B dwarfs. To this aim, we obtained and analyzed time series of high resolution $(\mathrm{R} \sim 46000)$, high $\mathrm{S} / \mathrm{N}$ spectra obtained with FIES@NOT in two observing runs. We applied the Fourier transform (Gray 1976) and the goodness-of-fit techniques to disentangle and measure the contributions from rotational $(v \sin i)$ and macroturbulent $\left(\Theta_{\mathrm{RT}}\right)$ broadening to the Si III 4567 and/or the O III 5592 line profiles. We quantified the LPVs in these lines by means of the first, $\langle v\rangle$, and third, $\left\langle v^{3}\right\rangle$, normalized velocity moments of the line. These moments are related to the centroid velocity and the skewness of the line profile, respectively, and are well suited to investigate whether an observed 

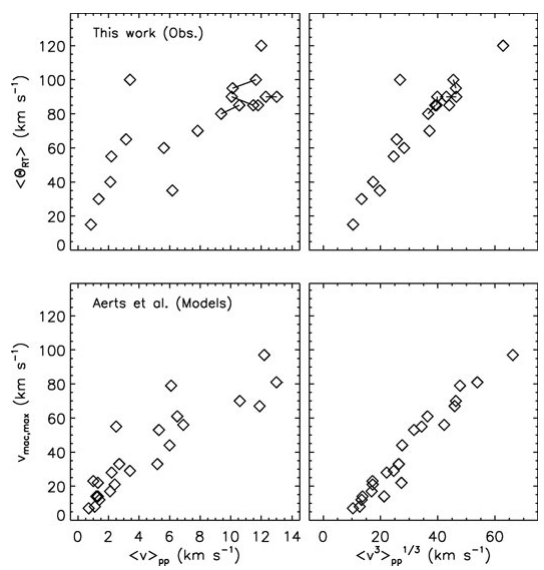

Figure 1. (Top) Empirical relations between the average size of the macroturbulent broadening $\left(\left\langle\Theta_{\mathrm{RT}}\right\rangle\right)$ and the peak-to-peak amplitude of the first and third moments of the line profile. Solid lines connect results from four stars observed in both campaigns. (Bottom) similar plots with data from Table 1 in Aerts et al. (2009), based on simulations of line profiles broadened by rotation and by hundreds of low amplitude non-radial gravity mode pulsations. The simulations lead to clear trends which are compatible with spectroscopic observations.

line profile is subject to time-dependent line asymmetry, as expected in the case of a pulsating star.

We found a clear positive correlation between the average size of the macroturbulent broadening, $\left\langle\Theta_{\mathrm{RT}}\right\rangle$, and the peak-to-peak amplitude of $\langle v\rangle$ and $\left\langle v^{3}\right\rangle$ variations (see Fig. 1). To our knowledge, this is the first clear observational evidence for a connection between extra broadening and LPVs in early $B$ and late $O$ Sgs.

\section{Is macroturbulent broadening in OB-Sgs caused by pulsations?}

Non-radial oscillations have been often suggested as the origin of LPVs and photosperic lines in OB Sgs; however, a firm confirmation (by means of a rigorous seismic analysis) has not been achieved yet. From a theoretical point of view, Saio et al. (2006) showed that g-modes can be excited in massive post-main sequence stars, as the g-modes are reflected at the convective zone associated with the H-burning shell. Lefever et al. (2007) presented observational evidence of g-mode instabilities in a sample of photometrically variable B Sgs from the location of the stars in the $\left(\log \mathrm{T}_{\text {eff }}, \log g\right)$-diagram.

These results, along with our observational confirmation of a tight connection between macroturbulent broadening and parameters describing observed LPVs render stellar oscillations the most probable physical origin of macroturbulent broadening in B Sgs; however, it is too premature to consider them as the only physical phenomenon to explain the unknown broadening.

\section{References}

Aerts, C., Puls, J., Godart, M., \& Dupret, M.-A. 2009, A\& A, 508, 409

Gray, D. F. 1976, The observation and analysis of stellar photospheres (New York: WileyInterscience), 1st Ed.

Lefever, K., Puls, J., \& Aerts, C. 2007, A\&GA, 463, 1093

Lucy, L. B. 1976, ApJ, 206, 499

Saio, H., Kuschnig, R., Gautschy, A., Cameron, C. et al. 2006, ApJ, 650, 1111

Simón-Díaz, S., Herrero, A., Uytterhoeven, K., Castro, N. et al. 2010, ApJ (Letters), 720, L174 